\title{
Reverse genetic screen for loss-of-function mutations uncovers a frameshifting deletion in the melanophilin gene accountable for a distinctive coat color in Belgian Blue cattle
}

\author{
Wanbo Li*1, Arnaud Sartelet ${ }^{\dagger 1}$, Nico Tamma*, Wouter Coppieters**, Michel Georges* and \\ Carole Charlier*
}

${ }^{*}$ GIGA-R and Faculty of Veterinary Medicine, University of Liège, 1, avenue de l'hôpital, 4000 Liège, Belgium. 'Bovine Clinic, FARAH and Faculty of Veterinary Medicine, University of Liège, 20, boulevard de Colonster, 4000 Liège, Belgium. *GIGA-Genomics platform, University of Liège, 1, avenue de l'hôpital, 4000 Liège, Belgium.

\section{Summary}

\begin{abstract}
In the course of a reverse genetic screen in the Belgian Blue cattle breed, we uncovered a 10-bp deletion (c.87_96del) in the first coding exon of the melanophilin gene (MLPH), which introduces a premature stop codon (p.Glu32Aspfs*1) in the same exon, truncating $94 \%$ of the protein. Recessive damaging mutations in the $M L P H$ gene are well known to cause skin, hair, coat or plumage color dilution phenotypes in numerous species, including human, mice, dog, cat, mink, rabbit, chicken and quail. Large-scale array genotyping undertaken to identify p.Glu32Aspfs*1 homozygous mutant animals revealed a mutation frequency of $5 \%$ in the breed and allowed for the identification of 10 homozygous mutants. As expression of a colored coat requires at least one wild-type allele at the co-dominant Roan locus encoded by the KIT ligand gene (KITLG), homozygous mutants for p.Ala227Asp corresponding with the missense mutation were excluded. The six remaining colored calves displayed a distinctive dilution phenotype as anticipated. This new coat color was named 'cool gray'. It is the first damaging mutation in the $M L P H$ gene described in cattle and extends the already long list of species with diluted color due to recessive mutations in MLPH and broadens the color palette of gray in this breed.
\end{abstract}

Keywords bovine, cool gray, disruptive mutation, KITLG, MLPH, OMIA 00206-9913, whole-genome/whole-exome sequence

In an attempt to evaluate the fraction of disruptive mutations that could cause embryonic lethality and therefore affect fertility, we embarked on a next-generationsequencing-based reverse genetic screen in modern cattle populations. As part of this study, we sequenced the whole genome of 50 and the whole exome of 30 sires from the Belgian Blue cattle breed. Sequence data were mined for highly disruptive loss-of-function variants corresponding to frameshift (FS), stop gain (SG) and essential donor/acceptor splice-site (SS) mutations. In this breed, we found 109 lossof-function variants, including 56 FS, 42 SG and 11 SS. We

Address for correspondence

C. Charlier, Unit of Animal Genomics, GIGA-R \& Faculty of Veterinary Medicine, University of Liège (B34), 1 Avenue de l'Hôpital, 4000-Liège, Belgium.

E-mail: carole.charlier@ulg.ac.be

${ }^{1}$ These authors contributed equally.

Accepted for publication 23 September 2015 have demonstrated that only a small fraction of these $(\sim 10 \%)$ likely causes embryonic lethality in homozygotes, highlighting the importance of molecular redundancy and the high proportion of non-essential genes (Charlier et al. 2014).

As a by-product of this study, we are currently searching for distinctive phenotypic features in animals that are apparently normal despite being homozygous for loss-offunction mutations in genes that are evolutionary highly conserved. In this process, we highlighted a 10-bp deletion in the first coding exon of the melanophilin gene $(M L P H)$ at genomic position $117,591,518-117,591,527 \mathrm{bp}$ on bovine chromosome 3 (BosTau6/UMD3.1 reference genome assembly) (Fig. 1). It is likely private to the Belgian Blue breed, as it was not listed in dbSNP (http:// www.ncbi.nlm.nih.gov/snp/), not found in the 1000 bull genomes project (http://www.1000bullgenomes.com) (run 3: 429 sequenced key ancestors from 15 different breeds) and not found in an additional panel of 10 breeds, including two bos indicus breeds. This FS mutation (c.87_96del) is 
(a)

(b)

\begin{tabular}{|c|c|c|c|c|c|c|c|c|}
\hline monewow & 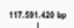 & 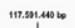 & insmenow on & 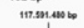 & 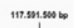 & 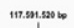 & 17msmsolo & 17n.sm.sonow \\
\hline
\end{tabular}

(c)

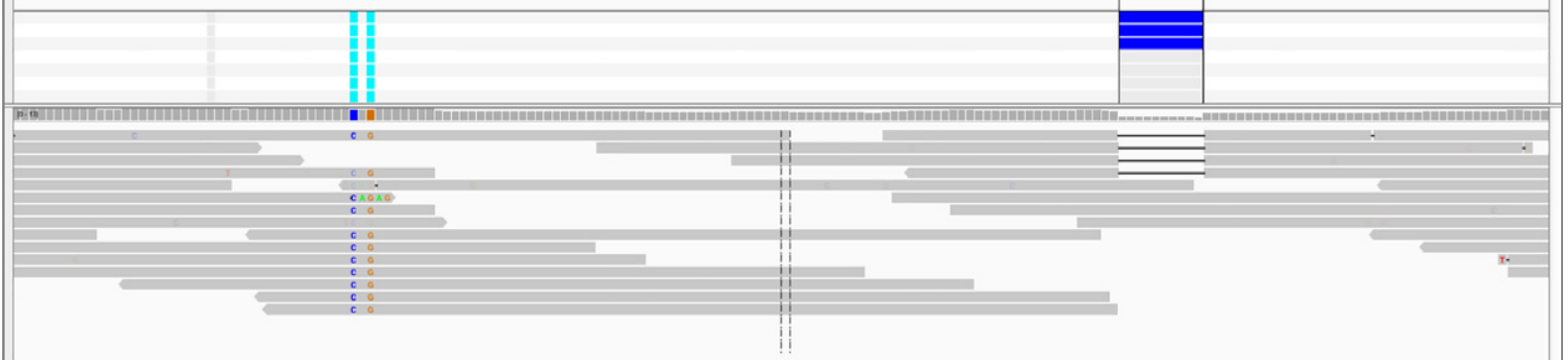

(d)

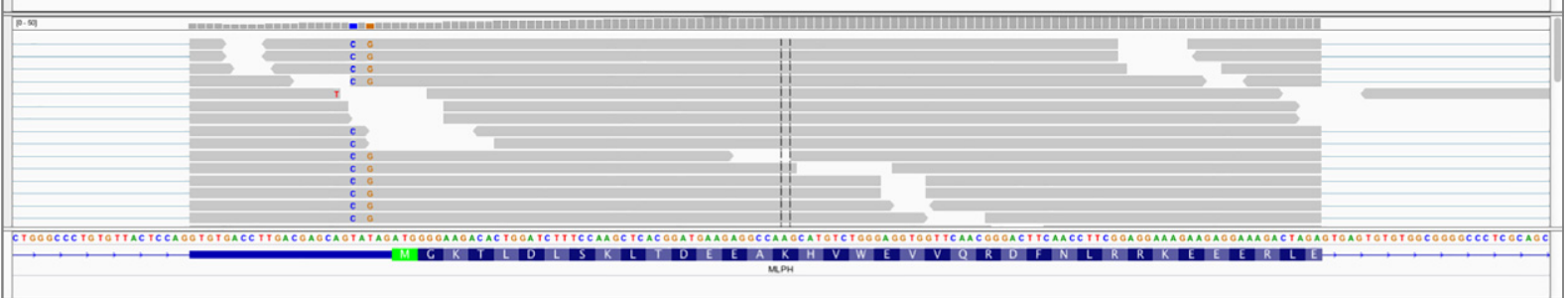

Figure 1 A 10-bp deletion (c.87_96del) in the first coding exon of the melanophilin gene. Screen capture of an INTEGRATIVE GENOMICS VIEWER (Robinson et al. 2011) output for a 182-bp genomic region encompassing the MLPH coding exon 1 with, from top to bottom (a) a 'VCF' file of 50 Belgian Blue sires (Druet et al. 2014) displaying three heterozygous animals for the c.87_96del mutation, (b) a 'BAM' file from a whole-genome sequence of a carrier animal, (c) fetal skin cDNA sequence reads from a wild-type animal defining the corresponding exon junctions and (d) a track of Ref-seq gene annotation.

predicted to introduce a premature stop codon in the same exon (p.Glu32Aspfs*1) and a resulting variant protein that is $94 \%$ shorter. Furthermore, the nonsense-mediated decay pathway is expected to degrade the variant mRNA.

The melanophilin gene encodes a protein expressed primarily in melanocytes, where it plays a central and active role in a tripartite complex (RAB27A-MLPH-MYO5A) (e.g. Strom et al., 2002; Sckolnick et al. 2013). This complex is indispensable for active intracellular mature melanosome trafficking. In human, mutations in any of the three subunits of the complex cause Griscelli syndrome, a recessive disorder characterized by variable immune and neurological defects systematically accompanied by skin and hair hypopigmentation. Mutations in $M L P H$ cause Griscelli syndrome type 3, which is strictly restricted to pigment dilution without any reported additional phenotypic change (Ménasché et al. 2003; Westbroek et al. 2012). This dilution is not generated by a defect in melanosome biosynthesis but, rather, caused by an impaired transport of mature melanosomes toward melanocyte dendritic tips where they are transferred to nearby keratinocytes (reviewed by Huizing et al. 2008). Similar hypopigmentation phenotypes, explained by recessive mutations in $M L P H$, have been described in numerous species including mouse (Matesic et al. 2001), cat (Ishida et al. 2006), dog (Philipp et al. 2005; Drögemüller et al. 2007), mink (Cirera et al. 2013), rabbit (Lehner et al. 2013; Fontanesi et al. 2014), chicken (Vaez et al. 2008) and quail (Bed'hom et al. 2012)but not yet cattle. The identified causative mutation(s) and associated dilution phenotype for each species are summarized in Table S1. Therefore, the c.87_96del variant in the bovine $M L P H$ gene appeared to be a strong candidate for a yet-to-be-described novel coat color in the Belgian Blue cattle.

The 109 loss-of-function variants detected in Belgian Blue cattle, including the MPLH:c.87_96del mutation, were added to the Illumina low-density custom array and used to genotype 5201 Belgian Blue animals as part of a genomic selection program (Charlier et al. 2014). The MLPH: c.87_96del mutation was shown to segregate at a frequency of $5 \%$ in Belgian Blue cattle and to be in HardyWeinberg equilibrium $(P=0.37 ; 10 \mathrm{del} / \mathrm{del}, 501 \mathrm{del} /+$, $4690+/+$ ). We identified 10 homozygous mutants. It is worth mentioning that the Belgian 'Blue' cattle breed received its name from the segregation of a co-dominant mutation, known as the Roan mutation, originating from the Shorthorn breed (Jones 1947). Homozygous wild-types, heterozygotes and homozygotes for the Roan mutation are black, roan blue (mixture of black and white hairs) and white, respectively (Charlier et al. 1996). These phenotypes are fully explained by a non-synonymous mutation (p.Ala227Asp; inferred annotation after gene model correction) in the KIT ligand (KITLG) gene (Seitz et al. 1999). This missense mutation was also present on our custom 
array and was shown to have a frequency of $56 \%$ in the breed. Among the $10 \mathrm{MLPH}$ homozygous mutants identified, two were black (KITLG:Ala227/Ala227), four roan blue (KITLG:p.Ala227Asp) and four white (KITLG:Asp227/ Asp227). As the phenotypic effect of the MLPH:c.87_96del mutation would be epistatically masked in white animals, we traced the six non-white animals back to their farms to examine their coat color. As expected, all six displayed a distinctive dilution phenotype, which we called 'cool gray' (Fig. 2, Appendix S1). Non-white animals, heterozygous for
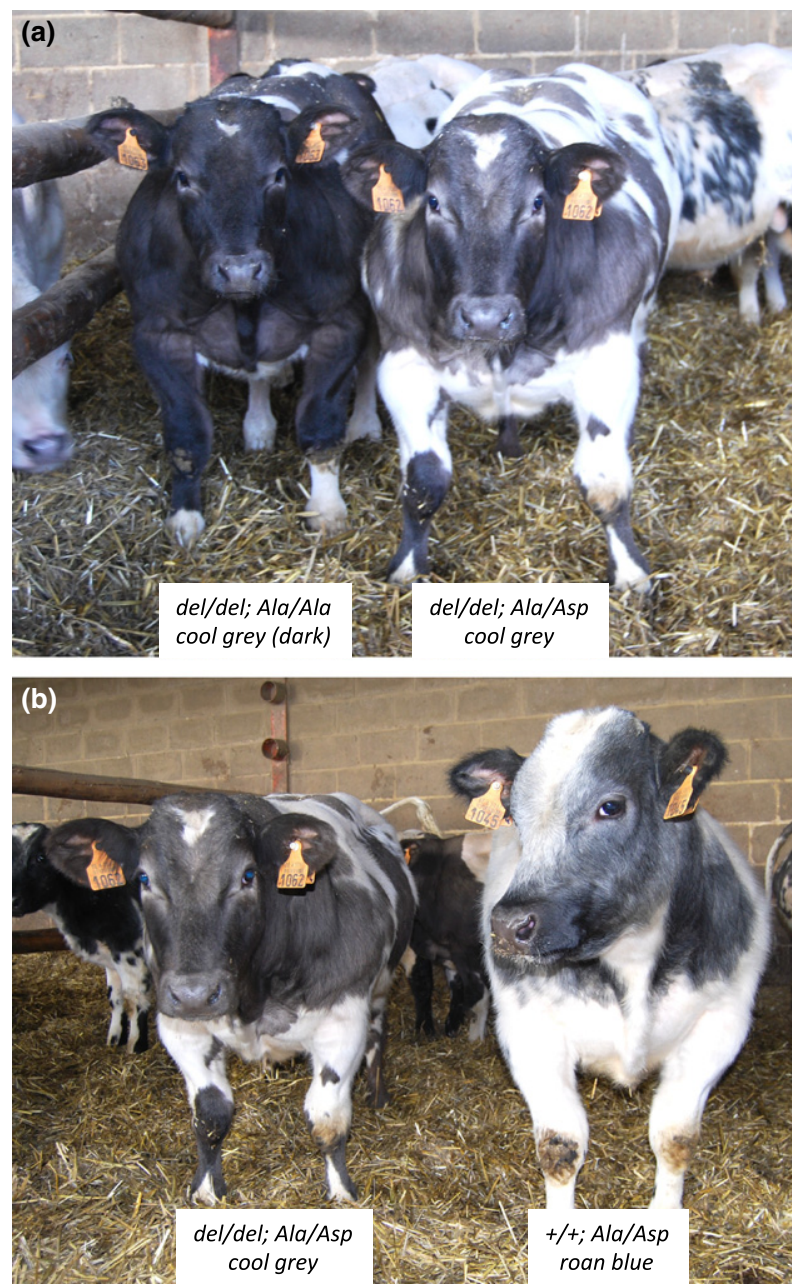

Figure $2 M L P H$ knockout dilution phenotypes in Belgian Blue animals and epistasis with the KITLG alleles. (a) Two animal homozygotes (del/ del) for the c.87_96del mutation at the MLPH locus: homozygote wildtype (Ala/Ala, left) and heterozygote (Ala/Asp, right) for the p.Ala227Asp non-synonymous mutation from the KITLG locus; the animal on the left displays a black dilute coat color (dark 'cool gray') and the one on the right shows the epistatic 'cool gray' coat color; however, distinguishing a phenotypic difference between these combinations of genotypes remains challenging when animals are not side by side. (b) Two animals heterozygotes (Ala/Asp) for the p.Ala227Asp non-synonymous mutation at the KITLG locus: homozygote mutant and wild type (del/del, left; +/+, right) for the c.87_96del mutation at the $M L P H$ locus; the animal on the right is under the classical 'roan blue' coat color. the MLPH:c.87_96del mutation, were indistinguishable from homozygote wild-type relatives (fully recessive mutation).

In all the species with loss-of-function mutations in $M L P H$, the reported phenotype appeared to be strictly restricted to skin and hairs. However, it is noteworthy that, in addition to melanocytes, $M L P H$ is also highly expressed in mast cells, which are key cellular players mediating allergic and inflammatory reactions. In mice, a recent study by Singh et al. (2013) has shown that the Rab27a/Mlph/ MyoVa complex seemed to regulate the docking of mast cell granules to the mast cell plasma membrane by modulating its cytoskeleton integrity. It is thus tempting to speculate that loss-of-function mutations in $M L P H$ could affect mast cell degranulation and have a pleiotropic effect on an allergic and/or inflammatory reaction's time-course. Moreover, in human, it has been reported that the MLPH locus exhibits a strong signal of recent positive selection in nonAfrican populations (Pickrell et al. 2009). A link between this sweep in human, underlying alleles and a putative advantageous phenotype-correlated or not with pigmentation-remains to be established.

Up until now, there have been characterized mutations segregating in the Belgian Blue breed at four coat-color loci (MC1R, KITLG, KIT and MLPH) (Klungland et al. 1995; Charlier et al. 1996; Seitz et al. 1999; Durkin et al. 2012; this study). All known mutations, their modes of inheritance, associated phenotypic effects and respective frequencies within this breed are listed in Table S2. Collectively, they are responsible for the observed variety of colored patterns and subtle blue or gray shades in both Belgian Blue purebred and crossbred animals.

This study stands as proof of the concept that a population-based next-generation-sequencing reverse screen can uncover segregating variations underlying novel phenotypes of biological interest.

\section{Acknowledgements}

We are grateful to Jean-Pierre Monfort and Gérard Bonduel (breeders) for their collaboration in cases collection and to the Walloon Breeding Association (AWE) for pedigree data. We also thank all the members of the GIGA-Genomic platform for their technical assistance. CC is Senior Research Associate of the Fonds National de la Recherche Scientifique (FNRS, Belgium). This work was funded by grants from the Walloon Ministry of Agriculture (Rilouke), the Belgian Science Policy Organisation (SSTC Genefunc PAI) and the University of Liège.

\section{Authors' contributions}

WL performed WGS/WES analysis; AS was in charge of cases collection and phenotyping; NT genotyped the samples on the Illumina custom array; WC supervised the 
GIGA-genomics platform; and MG and CC designed the study, analyzed the data and wrote the manuscript with the help of all co-authors.

\section{References}

Bed'hom B., Vaez M., Coville J.L., Gourichon D., Chastel O., Follett S., Burke T. \& Minvielle F. (2012) The lavender plumage colour in Japanese quail is associated with a complex mutation in the region of $M L P H$ that is related to differences in growth, feed consumption and body temperature. BMC Genomics 13, 442.

Charlier C., Denys B., Belanche J.I., Coppieters W., Grobet L., Mni M., Womack J., Hanset R. \& Georges M. (1996) Microsatellite mapping of the bovine roan locus: a major determinant of White Heifer disease. Mammalian Genome 7, 138-42.

Charlier C., Li W., Harland C., Littlejohn M., Creagh F., Keehan M., Druet T., Coppieters W., Spelman R. \& Georges M. (2014) NGSbased reverse genetic screen reveals loss-of-function variants compromising fertility in cattle. In: Proceedings of the 10th World Congress of Genetics Applied to Livestock Production, Vancouver, BC, Canada. Available at http://www.wcgalp.com/.

Cirera S., Markakis M.N., Christensen K. \& Anistoroaei R. (2013) New insights into the melanophilin $(\mathrm{MLPH})$ gene controlling coat color phenotypes in American mink. Gene 527, 48-54.

Drögemüller C., Philipp U., Haase B., Günzel-Apel A.R. \& Leeb T. (2007) A noncoding melanophilin gene (MLPH) SNP at the splice donor of exon 1 represents a candidate causal mutation for coat color dilution in dogs. Journal of Heredity 98, 468-73.

Druet T., Ahariz N., Cambisano N., Tamma N., Michaux C., Coppieters W., Charlier C. \& Georges M. (2014) Selection in action: dissecting the molecular underpinnings of the increasing muscle mass of Belgian Blue Cattle. BMC Genomics, 15, 796.

Durkin K., Coppieters W., Drögemüller C. et al. (2012) Serial translocation by means of circular intermediates underlies colour sidedness in cattle. Nature 482, 81-4.

Fontanesi L., Scotti E., Allain D. \& Dall'olio S. (2014) A frameshift mutation in the melanophilin gene causes the dilute coat colour in rabbit (Oryctolagus cuniculus) breeds. Animal Genetics 45, 248-55.

Huizing M., Helip-Wooley A., Westbroek W., Gunay-Aygun M. \& Gahl W.A. (2008) Disorders of lysosome-related organelle biogenesis: clinical and molecular genetics. Annual Review of Genomics and Human Genetics 9, 359-86.

Ishida Y., David V.A., Eizirik E., Schäffer A.A., Neelam B.A., Roelke M.E., Hannah S.S., O’Brien S.J. \& Menotti-Raymond M. (2006) A homozygous single-base deletion in MLPH causes the dilute coat color phenotype in the domestic cat. Genomics 88, 698-705.

Jones I.C. (1947) The inheritance of red, roan and white coat colour in dairy shorthorn cattle. Journal of Genetics. 48, 155-63.

Klungland H., Våge D.I., Gomez-Raya L., Adalsteinsson S.\& Lien S. (1995) The role of melanocyte-stimulating hormone (MSH) receptor in bovine coat colour determination. Mammalian Genome 6, 636-9.

Lehner S., Gähle M., Dierks C., Stelter R., Gerber J., Brehm R. \& Distl O. (2013) Two-exon skipping within MLPH is associated with coat color dilution in rabbits. PLoS One 8, e84525.

Matesic L.E., Yip R., Reuss A.E., Swing D.A., O'Sullivan T.N., Fletcher C.F., Copeland N.G. \& Jenkins N.A. (2001) Mutations in
Mlph, encoding a member of the Rab effector family, cause the melanosome transport defects observed in leaden mice. Proceedings of the National Academy of Sciences of the United States of America 98, 10238-43.

Ménasché G., Ho C.H., Sanal O., Feldmann J., Tezcan I., Ersoy F., Houdusse A., Fischer A. \& de Saint Basile G. (2003) Griscelli syndrome restricted to hypopigmentation results from a melanophilin defect (GS3) or a MYO5A F-exon deletion (GS1). The Journal of Clinical Investigation 112, 450-6.

Philipp U., Hamann H., Mecklenburg L., Nishino S., Mignot E., Günzel-Apel A.R., Schmutz S.M. \& Leeb T. (2005) Polymorphisms within the canine $M L P H$ gene are associated with dilute coat color in dogs. BMC Genetics 6, 34.

Pickrell J.K., Coop G., Novembre J. et al. (2009) Signals of recent positive selection in a worldwide sample of human populations. Genome Research 19, 826-37.

Robinson J.T., Thorvaldsdóttir H., Winckler W., Guttman M., Lander E.S., Getz G. \& Mesirov J.P. (2011) Integrative genomics viewer. Nature Biotechnology 29, 24-6.

Sckolnick M., Krementsova E.B., Warshaw D.M. \& Trybus K.M. (2013) More than just a cargo adapter, melanophilin prolongs and slows processive runs of myosin Va. Journal of Biological Chemistry 288, 29313-22.

Seitz J.J., Schmutz S.M., Thue T.D. \& Buchanan F.C. (1999) A missense mutation in the bovine MGF gene is associated with the roan phenotype in Belgian Blue and Shorthorn cattle. Mammalian Genome 10, 710-2.

Singh R.K., Mizuno K., Wasmeier C., Wavre-Shapton S.T., Recchi C., Catz S.D., Futter C., Tolmachova T., Hume A.N. \& Seabra M.C. (2013) Distinct and opposing roles for Rab27a/Mlph/MyoVa and Rab27b/Munc13-4 in mast cell secretion. FEBS Journal 280, 892-903.

Strom M., Hume A.N., Tarafder A.K., Barkagianni E. \& Seabra M.C. (2002) A family of Rab27-binding proteins. Melanophilin links Rab27a and myosin Va function in melanosome transport. Journal of Biological Chemistry 277, 25423-30.

Vaez M., Follett S.A., Bed'hom B., Gourichon D., Tixier-Boichard M. \& Burke T. (2008) A single point-mutation within the melanophilin gene causes the lavender plumage colour dilution phenotype in the chicken. BMC Genetics 9, 7 .

Westbroek W., Klar A., Cullinane A.R. et al. (2012) Cellular and clinical report of new Griscelli syndrome type III cases. Pigment Cell and Melanoma Research 25, 47-56.

\section{Supporting information}

Additional supporting information may be found in the online version of this article.

Table S1 Known recessive mutations in the MLPH gene and associated effect at the molecular level in various vertebrate species.

Table S2 Known mutations and their associated phenotypic effect in the four coat color genes molecularly characterized in the Belgian Blue breed.

Appendix S1 Photographs of Belgian Blue animals and genotype/phenotype table. 